\title{
Modeling the transition between stable and unstable operation while emptying a silo
}

\author{
J. S. Leszczynski · T. Blaszczyk
}

Received: 28 September 2009 / Published online: 25 December 2010

(C) The Author(s) 2010. This article is published with open access at Springerlink.com

\begin{abstract}
In this paper we have examined the stable and unstable states of the operation during the emptying of the silo. Here the state of stable operation of the silo is understood as a smooth mass outflow. The unstable state is manifested by funnel flow or arching. This issues from a moistured granular material. We have focused on the two extreme experimental cases. The first option was considered as a mass flow and the second one was considered as arching. Based on experimental data we have simulated the silo emptying by DEM. Considering the transition between stable and unstable operation we proposed a novel mathematical model of the silo emptying. This model involves a fractional-differential oscillator equation. Analyzing the solution of this equation we have presented how to recognize the state of stable and unstable operation during silo emptying.
\end{abstract}

Keywords Silo emptying - Mass flow · Funnel flow · Arching $\cdot$ DEM $\cdot$ Fractional calculus .

Fractional differential equation

\section{Introduction}

The problem of continuous operation of silos, due to the behavior of granular material inside the silo, is still a challenge for industrial and scientific communities. This is a very

\section{J. S. Leszczynski ( $ه)$}

Department of Heating, Ventilation and Air Protection,

Czestochowa University of Technology, ul. Dabrowskiego 73,

42-200 Czestochowa, Poland

e-mail: jaclesz@gmail.com

\section{T. Blaszczyk}

Institute of Mathematics, Czestochowa University of Technology, ul. Dabrowskiego 73, 42-200 Czestochowa, Poland

e-mail: tomblaszczyk@gmail.com interesting topic and, therefore, arises a lot of experimental and theoretical studies $[6,8,12,26,29,34]$. Examining the work during the emptying of the silo can be generally seen a mass outflow of granular material or funnel flow, or arching. During the mass outflow the granular material flows in a smooth manner from the silo under the action of gravity. In this case one can observe the linear loss of mass outflow over time. However, the impact of the construction of a silo, for example, inclined angle of the hopper, tha wall roughness, or the impact of processes occurring within the granular material, such as capillary cohesion change occur in the flow pattern which are much less well understood. Therefore one can observe funnel flow, where most of the motion occurs in a central core and near the walls stagnant regions of granular flow is noted [30]. In this case one can observe the nonlinear loss of mass outflow over time. Sometimes, however, there is a lack outflow of granular material from the silo. In this case the flow is stoped and the granular material forms an arc surface in different heights of the hopper [37]. Referring to the mass outflow it should be noted that this flow is the result of proper inclined angle of the hopper, the wall smoothness, and gravity domination over internal processes occuring in particle-particle and particle-wall contacts. However, if any of these conditions is not fulfilled the mass flow changes to funnel flow. External observations of the dynamics of emptying a silo can not recognize what type of outflow dominates. In the literature $[33,36,37]$ there is a series of processes and phenomena associated dynamics of emptying the silo, which can be conventionally divided into: arching, ratholing, irregular flow, wide resident distribution, jamming, segregation, etc. In the phenomenological point of view, the process of emptying of the silo attempts to explain using anomalous diffusion [3]. Through analysis of velocity and concentration profiles of particles [3] one can seen superdiffusion behavior in granular material flow from the 
silo. In the literature $[3,5,6,15,22,23,27]$ one can find a lot of studies in which the authors propose different approaches to the mathematical description of the dynamics of the outflow of granular material from the silo. They have certain limitations especially when we consider granular cohesion dynamics. Taking into account the results presented in [3] we propose a new mathematical description of the process of emptying the silo. On the basis of fractional calculus [16] we propose an ordinary-fractional differential equation that must satisfy two basic conditions: the initial condition (a silo is full) and the final condition (the silo is empty). The ordinary differential equation is called the fractional oscillator equation. This equation follows from the principle of minimum action and fractional integration by parts [1].

\section{Experimental investigation and DEM simulations}

Some simple experimental tests made in order to clarify the transition from stable to unstable silo operations. These studies have been devoted to the influence of the size of the capillary forces on the process of emptying a silo. Figure 1 shows the basic dimensions of the silo. Experimental studies were carried out for 3,000 particles of peas with the following parameters: density $\rho_{p}=1,300 \mathrm{~kg} / \mathrm{m}^{3}$, Young's modulus $E=4 \times 10^{6} \mathrm{~Pa}$, Poisson's ratio $v=0.2$, particle size distribution described by Rosin-Rammler distribution $d_{63}=$ $0.0071 \mathrm{~m}$ and $n=18$. In order to estimate the amount of capillary cohesion we made some shear tests. In [35] presented some interesting experience in shear tests of food particles. Here, tests ware made in the Division of Soil-Structure Interaction at Cracow University of Technology, Poland. The shear tests performed in the direct/residual shear machine 27-T2160 [9]. The experiment was performed for two cases. The first case considered dry pea particles which include natural moisture with the amount of $11.06 \%$. The second one focused on the moist particles with the water content about $27.40 \%$. In the first case we performed four shear tests for different loads of $25,50,75,100 \mathrm{~kg}$. For wet pea particles made only three shear tests for the following loads 25,50 , $100 \mathrm{~kg}$. Figure 2 shows results of the shear tests. Above figure presents shear over normal stresses for different shear tests. Analyzing such results one can say that capillary cohesion $c$ is predicted by the internal friction angle $\varphi$. For natural amount of moisture $11.06 \%$ represented by circles, we estimated the internal friction angle $42.7^{\circ}$ and the capillary cohesion was $c=5.5 \mathrm{kPa}$. However, for moist particles indicated by asterisks we found $\varphi=39.1^{\circ}$ and $c_{\max }=10.6 \mathrm{kPa}$. Next we add dashed line which represents approximate behaviour of granular material without any moisture. This state applied for DEM simulations. According to values of the internal friction angle we calculated coefficient of static friction $\mu_{s t}=0.92$

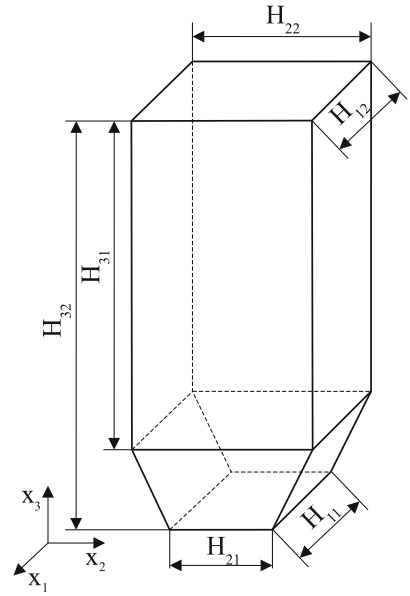

Fig. 1 Silo dimensions adopted in the experiment: $H_{11}=H_{21}=$ $0.0425 \mathrm{~m}, H_{22}=H_{12}=0.073 \mathrm{~m}, H_{31}=0.299 \mathrm{~m}, H_{32}=0.399 \mathrm{~m}$

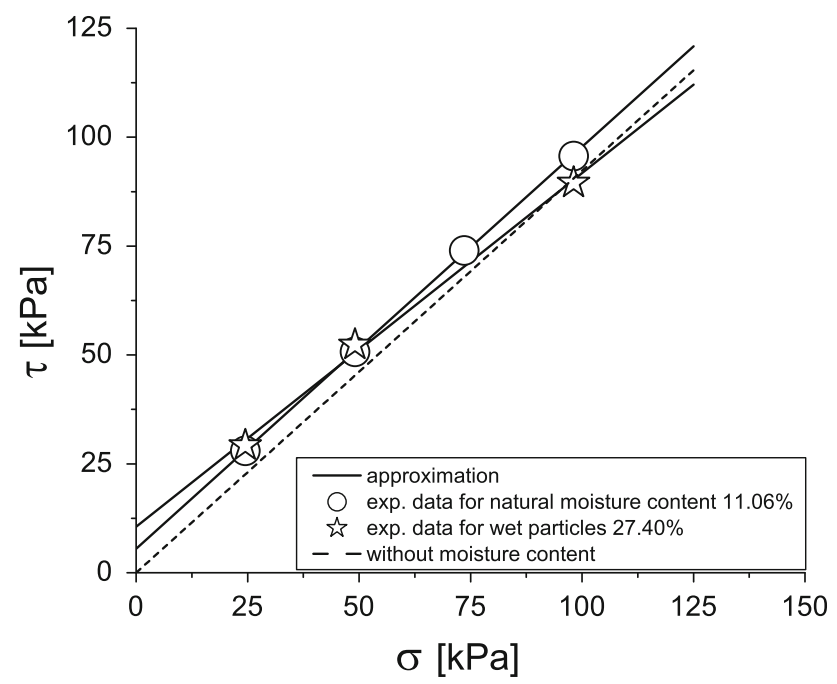

Fig. 2 Experimental results of direct shear tests for dry and wet pea particles

for dry pea particles and $\mu_{s t}=0.81$ for the wet ones. These values are necessary to calibrate DEM calculations.

Next we considered an outflow of pea particles from a silo. Therefore we used the container with dimensions presented by Fig. 1. The experiment was performed for two cases. The first case concerned an outflow of dry particles. The second one concerned an outflow of moist particles. As a basic operating parameters of the silo adopted the begining height formed by the peas $h_{\text {bed }}$ and time emptying the silo with granular material $T_{d}$. The emptying time $T_{d}$ was recorded by a digital camera with an accuracy of $\pm 0.04 \mathrm{~s}$. Figure 3 presents the initial states of granular material filling the silo. The lock located in the bottom part of silo is discharged when the silo was filled with granular material. During the outflow of granular material from the silo emptying time $T_{d}$ was measured by the digital camera. In the first case presented in Fig. 3a, when the granular material 
Fig. 3 Two initial states of granular material filling the silo: a 3,000 of dry pea particles, $h_{\text {bed }}=0.21 \mathrm{~m}, \mathbf{b} 3,000$ of moist pea particles, $h_{\text {bed }}=0.23 \mathrm{~m}$
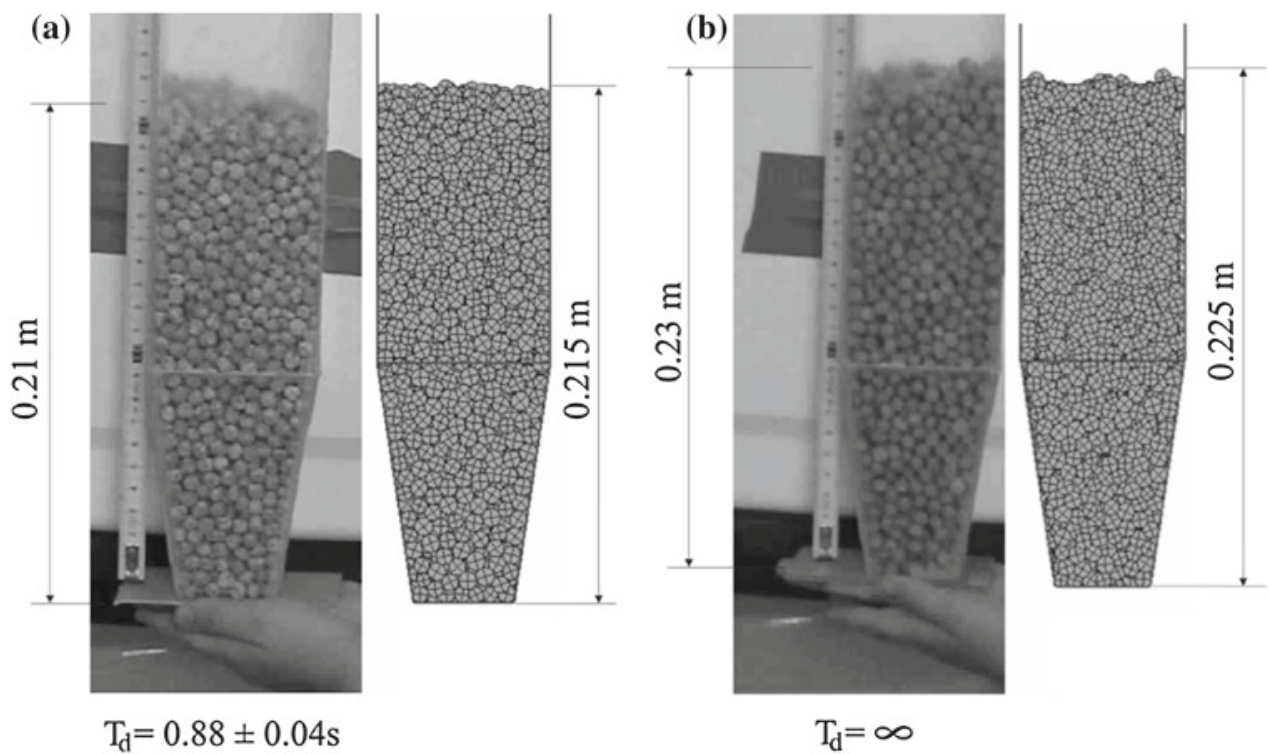

was dry, there was a smooth outflow of particles from the silo. One can say that in this case we are dealing with a mass outflow. The total duration time of emptying the silo was $T_{d}=0.88 \pm 04 \mathrm{~s}$. In the second case shown in Fig. $3 \mathrm{~b}$, when the granular material has a certain amount of moisture represented by capillary cohesion $c_{\max }=10.6 \mathrm{kPa}$, there was a blockade of particles in a silo. Outflow of particles has been stopped. They have formed an area of the curve at the bottom of the silo hopper. The blockade of the outflow occurred as a result of capillary action of strong liquid bridges between contacting pairs of particles and between contacting particles with the silo wall. It should be noted that in both these cases we are dealing with extreme states. In the first case there is almost no capillary forces as a result of natural moisture in the granular material. The second case, where introduced a certain amount of water to the granular medium, shows how strong may be the capillary forces.

With regard to Jenike theory [14], we estimated the critial (smallest) outlet size (with or diameter) of the hopper in order to indicate arching. Here we used the calculation procedure which was presented by Drescher et al. [10,11] and Schulze [34]. Table 1 shows calculation results for the silo outlet, where particles with different moisture conntent were used. In the table, $\varphi$ is the internal friction angle, $\delta$ represents the effective friction angle, $\varphi_{w}$ is the wall friction angle, $\rho_{b}$ is the bulk density, $\gamma$ denotes the bulk unit weight, $\sigma_{1}$ is the major principal stress, $\sigma_{c s}$ represents the compressive stress in the arch and $\sigma_{c p}$ is the uniaxial compressive yield stress. We assumed the flow factor $f f$ according to Arnold and McLean theory [4] in order to calculate the stresses. It should be noted that all values of stresses were determined in the square outlet $b_{s i}=4.25 \mathrm{~cm}$ of the silo (Fig. 1). The height from the hopper apex to the silo outlet was $h_{s i}=13.9 \mathrm{~cm}$. According to data presented by Table 1 and the calculation procedure of the minimum size of the outlet [34] we obtain the critial outlet size. Figure 4 illustrates the major principal stress $\sigma_{1}$, the compressive stress in the $\operatorname{arch} \sigma_{c s}$ and the uniaxial compressive yield stress $\sigma_{c p}$ which are proportional to the local hopper outlet. In turn, the local hopper outlet is proportional to the distance $h$, to the virtual hopper apex. In making the calculations, we received the following sizes of outlets: $b_{\text {crit }}=2.48 \mathrm{~cm}$ for natural wet particles with the amount of moisture content $11.06 \%$ and $b_{\text {crit }}=5.90 \mathrm{~cm}$ for the moisture content $27.40 \%$. In comparison to the silo outlet $b_{s i}=4.25 \mathrm{~cm}$ (Fig. 1) we noticed $b_{c r i t}>b_{s i}$ for $11.06 \%$ of moisture content and $b_{c r i t}<b_{s i}$ for the wet particles with $27.40 \%$ of moisture content. This means that for small amount of moisture content in particles we expect the mass outflow. However, the arch occurs for the larger moisture content in granular material. It should be noted that for all dry particles we received $\sigma_{c s}=0$, because in this case $\varphi=\delta$.

For a more detailed analysis of the outflow of granular material, we are interested in the states which are intermediate states between those cases. It should be emphasized that knowledge of the behavior of granular material in the intermediate states will determine the moment in which the blockade of the silo occurs. However, to carry out such a series of experiments is technically complicated and requires the involvement of advanced research equipment. Therefore, using DEM [25] we decided to carry out simulations of intermediate states of the outflow of granular material from the silo. Simulations were performed for the same set of parameters describing the granular material as in the experiment. However, we neglect natural cohesion for dry particles. In addition, we have assumed coefficient of static friction $\mu_{s t}=$ 0.92 for dry pea particles and $\mu_{s t}=0.81$ for wet ones. These values are taken from the experiment carried out previously. 
Table 1 Pea parameters for calculations of stresses in the silo outlet

\begin{tabular}{lllllllll}
\hline Material & $\varphi\left(^{\circ}\right)$ & $\delta\left(^{\circ}\right)$ & $\varphi_{w}\left({ }^{\circ}\right)$ & $\rho_{b}\left(\mathrm{~kg} / \mathrm{m}^{3}\right)$ & $\gamma\left(\mathrm{kN} / \mathrm{m}^{3}\right)$ & $\sigma_{1}(\mathrm{kPa})$ & $\sigma_{c s}(\mathrm{kPa})$ & $\sigma_{c p}(\mathrm{kPa})$ \\
\hline Dry $w=0 \%$ & 43.00 & 43.00 & 21.0 & 741.5 & 7.27 & 0.461 & 0.312 \\
Natural wet $w=11.06 \%$ & 42.70 & 44.29 & 21.0 & 851.6 & 8.35 & 0.520 & 0.358 \\
Wet $w=27.40 \%$ & 39.10 & 42.37 & 18.0 & 1015.0 & 9.96 & 0.690 & 0.427 & 0.271 \\
\hline
\end{tabular}

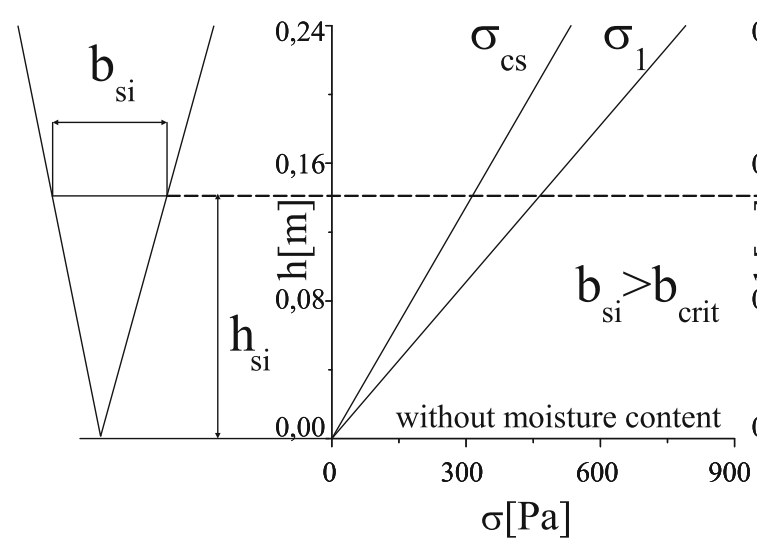

Fig. 4 Determination of critical outlet size $b_{\text {crit }}$ to avoid arching

Next we have assumed coefficient of kinetic friction $\mu_{d y}=$ 0.05 , surface tension for wet particles $\gamma=0.0725 \mathrm{~J} / \mathrm{m}^{2}$. In order to reduce computational time we assumed nonlinear interaction law [24] as a model of repulsive force. However, the model of repulsive force requires the correct estimation of the coefficients of elasticity $\tilde{k}_{i, j}$ and damping $\tilde{c}_{i, j}$ in order to reflect the actual energy dissipation and contact time during particle-particle and particle-wall collisions. It should be noted that the coefficient of elasticity $\tilde{k}_{i, j}$ is a constant value for arbitrary materials being in contact. It can be estimated by the following formula

$\tilde{k}_{i, j}=\frac{\frac{4}{3} \sqrt{\tilde{r}_{p_{i, j}}}}{\frac{1-v_{i}^{2}}{E_{i}}+\frac{1-v_{j}^{2}}{E_{j}}}$

where $\tilde{r}_{p_{i, j}}=\frac{r_{p_{i}} r_{p_{j}}}{r_{p_{i}}+r_{p_{j}}}$ and $v_{i}, v_{j}$ are Poisson's ratios, and $E_{i}$, $E_{j}$ represent Young's moduluses. However, we assumed the damping coefficient $\tilde{c}$ as

$\tilde{c}_{i, j}=2 H_{\tilde{c}_{i, j}} \sqrt{\frac{\tilde{m}_{p_{i, j}} \tilde{k}_{i, j}}{\sqrt{\tilde{r}_{p_{i, j}}}}}$

where $\tilde{m}_{p_{i, j}}$ is a reduced mass of contacting bodies and $H_{\tilde{c}_{i, j}}$ is an empirical constant. In order to predict the empirical constant $H_{\tilde{c}_{i, j}}$ we performed preliminary simulations for a stack of particles which contact with the bottom plate due to gravity action. In these simulations we used the fractional interaction law [25] as a model of repulsive force which gives proper results in the energy dissipation and contact time for multiparticle collisions. For above data we estimated the empirical constant as $H_{\tilde{c}_{i, j}}=0.1$.

According to [28] we assumed the form of capillary force acting between two particles as

$P_{i, j}^{L B}=\pi \gamma \tilde{r}_{p_{i, j}} \frac{r_{i, j}^{I I}\left(r_{i, j}^{I}+r_{i, j}^{I I}\right)}{r_{i, j}^{I I}}$

where $\tilde{r}_{p_{i, j}}=\frac{r_{p_{i}} r_{p_{j}}}{r_{p_{i}}+r_{p_{j}}}$ and

$r_{i, j}^{I}=\frac{1+Z_{j}-\cos \left(\psi_{j}\right)}{\cos \left(\psi_{j}\right)}$

$r_{i, j}^{I I}=\frac{\left(1+Z_{i, j}\right)\left(\sin \left(\psi_{i, j}\right)-1\right)+\cos \left(\psi_{i, j}\right)}{\cos \left(\psi_{i, j}\right)}$

are functions of the wetting angle $\psi_{i, j}$ and

$Z_{i, j}=\max \left[0,-\frac{\left\|\zeta_{i, j}\right\|}{2 \tilde{r}_{p_{i, j}}}\right]$

However, the wetting angle is calculated through solution of the following equation 


$$
\begin{aligned}
f\left(\psi_{i, j}\right)= & \frac{3}{2}\left(\left(r_{i, j}^{I}\right)^{2}\left(\left(1+Z_{i, j}\right)-\left(r_{i, j}^{I}+r_{i, j}^{I I}\right)\right)\right. \\
& \left.-\frac{Z_{i, j}^{2}\left(Z_{i, j}+3\right)}{3}\right)-\Phi_{i, j}=0
\end{aligned}
$$

where $\Phi_{i, j}=\frac{V_{p_{i, j}}^{L B}}{\tilde{V}_{p_{i, j}}}$ is quotient of the volume of the liquid layer surrounding particles $V_{p_{i, j}}^{L B}$ which is in relation to particle volumes $\tilde{V}_{p_{i, j}}=V_{p_{i}}+V_{p_{j}}$. It should take into considerations that the wetting angle belongs to range $\psi_{i, j} \in\left\langle 0, \frac{\pi}{2}\right)$.

Correct estimation of all coefficients included in DEM is one of the necessary condition to carry out simulations which are consistent with experimental data. Since no measurement of the wall stresses were performed during experiment, the comparison has been made between DEM results and calculations performed by theory. The stresses in the silo at discharge state are calculated due to procedure proposed by Arnold and McLean [4]. In this procedure the theory of Jenike [14] is used and the wall stresses were performed starting from Janssen slice element method [13]. Figure 5 shows a comparison of profiles of wall stresses $\sigma$ calculated by DEM and theory. Analyzing the behavior of the wall stresses one can observe poor agreement between DEM simulations and the Jenike theory in the top part of the silo. This difference results from an averaging thechnique used in DEM for calculations the wall stresses and the bulk density assumed in the Jenike theory. Nevertheless, in the hopper we observe good agreement between DEM simulations and the theory. Within such calculations we paid special attention in determining the stress peak at the transition between wedge-shaped hopper and silo.

With regard to DEM, we performed simulations of two extreme cases which have been investigated experimentally. Figure $3 a$ and $b$ represent the initial heights of layers being a result of preliminary simulations of granular material filling the silo. For dry particles we note $h_{\text {bed }}=0.215 \mathrm{~m}$ and for wet ones we have $h_{\text {bed }}=0.225 \mathrm{~m}$. Both results are slightly different from the amount determined in the experiment. This is due to the model assumptions such as: particles are perfectly spherical, particle distribution is described by the RosinRammler function, etc. However, the discrepancy between the model and experiment in determining the amount of layers is small. Assuming the above initial conditions we tried to simulate the dynamics of emptying the silo for two extreme cases referred by the experiment. In numerical computations we assumed time step $\Delta t=2 \times 10^{-5} \mathrm{~s}$. Figure 6 shows a comparison of the simulation dynamics emptying a silo with experimental data for the first case presented by Fig. 3a. We have seen that in the case of the experiment total time of emptying the silo was $T_{d}=0.88 \pm 0.04 \mathrm{~s}$. By contrast, for the simulation the total duration of emptying a silo was equal to $T_{d}=0.86 \pm 0.04 \mathrm{~s}$. Comparing the total times of

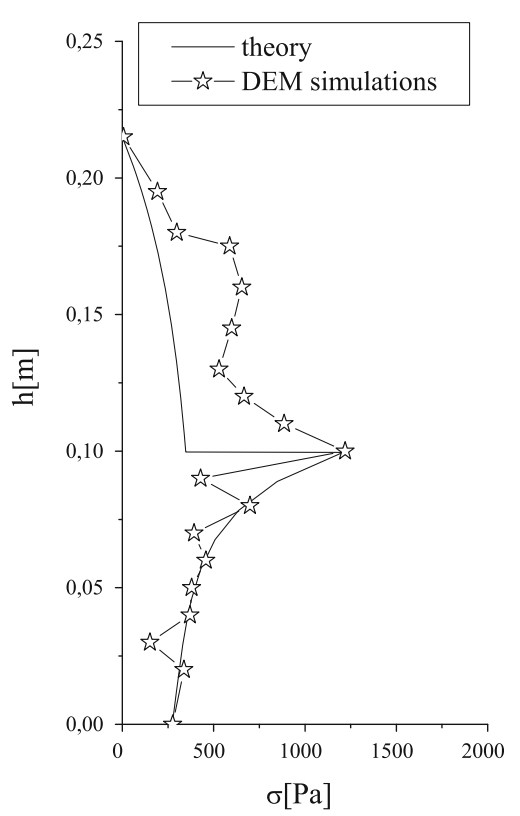

Fig. 5 Comparison of wall stresses calculated for dry pea particles

emptying of the silo can be said that sufficient compatibility of the results obtained from the simulation and experimental data. Moreover, for the second case (wet particles) we ware also carried out a computer simulation. For this case we got similar results as for the experiment, i.e. the granular material remains at all times in a silo. Having thus verified experimentally the DEM we focused on further simulations. Given the simulation results for dry particles, our research consisted in the gradual increase of the capillary force from zero to the extreme value that was determined by the second case-wet particles. In this way, we obtained results that show the transition from the stable operation of the silo to its unstable state. Section 4 presents the results of these simulations.

\section{Mathematical modeling}

With regard to fractional differential equations, we note that in recent years begun to explore the equations containing composition of fractional derivatives, i.e. the left composed with the right-sided derivative or vice versa. These equations appear in the fractional theoretical mechanics, which applies the principle of minimum action and fractional integration by parts [1]. Riewe [31,32] was the first who obtained such equations. By contrast, Klimek $[17,18]$ and Agrawal $[1,2]$ proposed a slightly different approach to obtain such a class of equations. The common feature of factional equations of this type is mutual relationship both left- and right-sided fractional derivatives, even if any of output action depends only on one of them. In work [3] has confirmed the thesis that 

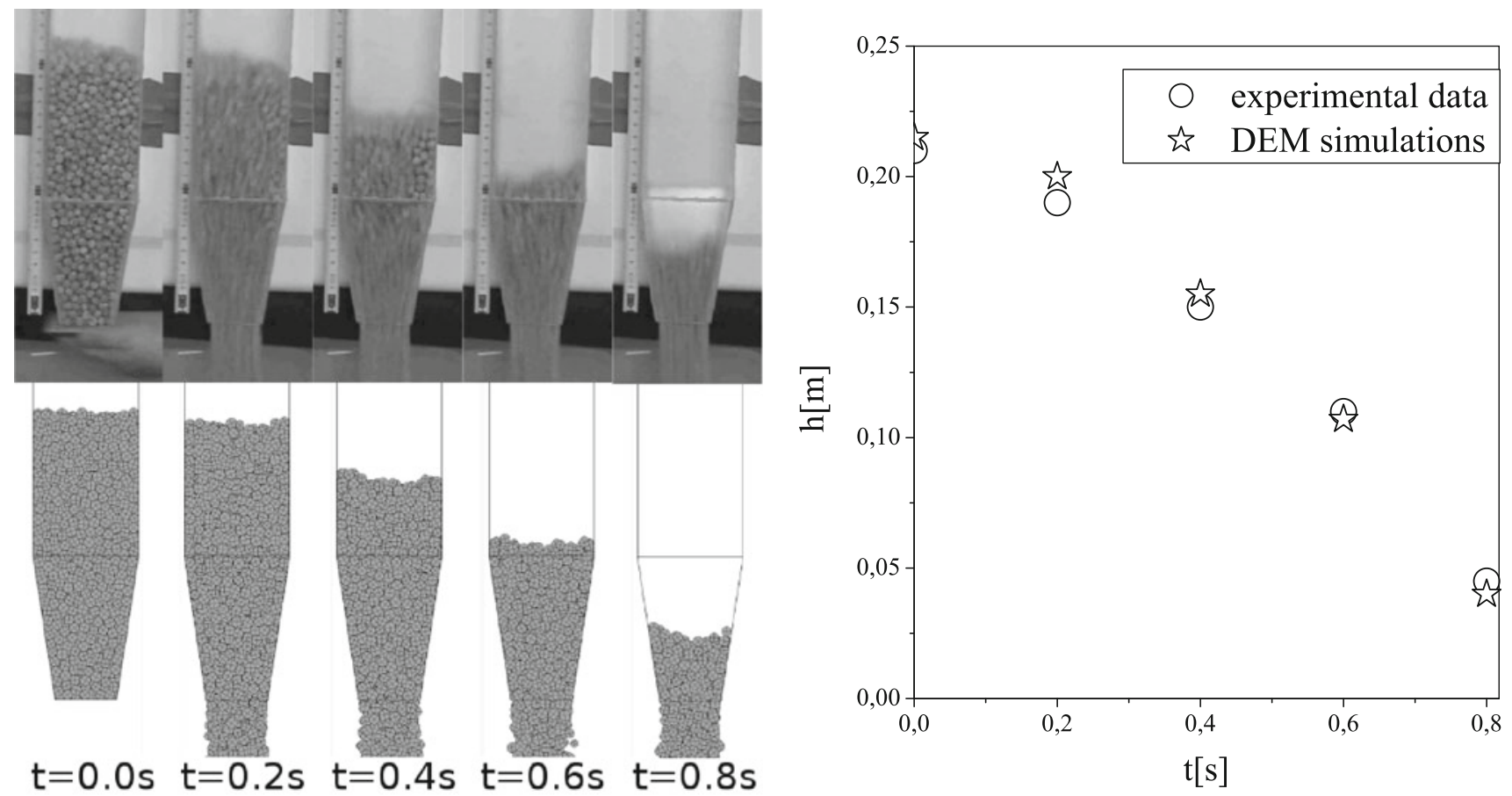

Fig. 6 Comparison of simulation emptying a silo with experimental data for the first case-dry particles

features describing the outflow of granular material during the emptying of the silo are superdiffuse.

Against this background, we consider a fractional differential equation describing the height loss over time of the granular material contained in a silo. According to $[1,2,17$, $18]$ we propose a fractional oscillator equation as

${ }^{C} D_{T-}^{\alpha} D_{0+}^{\alpha} h^{*}(t)+\beta h^{*}(t)=0, \quad t \in[0, T]$

where $h^{*}(t)=h_{\text {bed }}-h(t)$ represents an investigated function, $h_{\text {bed }}$ is the initial bed height, $h(t)$ is the height loss of the granular bed due to silo emptying, and $D_{0+}^{\alpha} h^{*}(t)$ indicates the left-sided Riemann-Liouville fractional derivative [16] which is defined as

$D_{0+}^{\alpha} h^{*}(t)=\frac{1}{\Gamma(1-\alpha)} \frac{d}{d t} \int_{0}^{t} \frac{h^{*}(s) d s}{(t-s)^{\alpha}}$

and ${ }^{C} D_{T-}^{\alpha} h^{*}(t)$ represents the right-sided Caputo fractional derivative [16] which its form is

${ }^{C} D_{T-}^{\alpha} h^{*}(t)=\frac{1}{\Gamma(1-\alpha)} \int_{t}^{T} \frac{\left(h^{*}\right)^{\prime}(s) d s}{(s-t)^{\alpha}}$

In Eqn. (8) $\alpha$ is non-integer order of the equation and $\beta$ represents an arbitrary constant coefficient. In order to clarify how this fractional oscillator equation works, we consider the situation for which the non-integer order of the equation tends to unity, i.e. $\alpha \rightarrow 1$. In this case Eqn. (8) becomes the classical oscillator equation
$D^{2} h^{*}(t)+\beta h^{*}(t)=0$

Therefore, we limit the non-integer order to the interval $\alpha \in$ $(0,1)$. Klimek in $[19,20]$ presented the analytical solution of Eqn. (8). The function $h^{*}(t)$ in a limited period of time is a series of integrals [21], which takes the form

$h^{*}(t)=\sum_{m=0}^{\infty} \beta^{m}\left(I_{0+}^{\alpha} I_{T-}^{\alpha}\right)^{m}\left[\sum_{k=-n+1}^{n-1} c_{k} t^{\alpha+k}\right]$

where

$I_{0+}^{\alpha} h^{*}(t):=\frac{1}{\Gamma(\alpha)} \int_{0}^{t} \frac{h^{*}(s) d s}{(t-s)^{1-\alpha}} \quad t>0$

and

$I_{T-}^{\alpha} h^{*}(t):=\frac{1}{\Gamma(\alpha)} \int_{t}^{T} \frac{h^{*}(s) d s}{(s-t)^{1-\alpha}} \quad t<T$

represent left- and right-sided fractional integral operators which are called Riemann-Liouville fractional integrals [16]. Moreover $c_{k}$ are arbitrary constants and $n=[\alpha]+1$ where $[\cdot]$ indicates an integer part of real number. The solution Eqn. (12) has a complex form, therefore it is difficult to apply, for example to do a chart. Therefore, we will seek a numerical solution of Eqn. (8). In the paper [7] we proposed such a numerical scheme. However, we want to introduce this scheme here and adapt it to our equation form. 
Let $t \in[0, T]$ then the interval is divided into $N$ parts, so that $0=t_{0}<t_{1}<t_{2}<\cdots<t_{N}=T$, where it is assumed constant time step $\Delta t=t_{i+1}-t_{i}$, for $i=0 \ldots N-1$. Let us denote $h_{k}^{*}=h^{*}\left(t_{k}\right)$, for $k=0 \ldots N$. Then we can enter the initial and final conditions, which exist at the ends of the interval $[0, T]$. Given the specific nature of left-sided derivative Eqn. (9) we assumed the initial value of the function $h^{*}(t)$ to be zero. While the final condition can be any real number. Thus we have

$$
h^{*}(0)=0 \quad h^{*}(T)=h_{\text {bed }}
$$

Taking into account the above assumptions and $k=1, \ldots$, $N-1$, we obtain a discrete form of Eqn. (8) as

$$
\Delta t^{-2 \alpha} \sum_{i=k}^{N}\left[w(k, i) \sum_{j=0}^{i} v(i, j) h_{j}^{*}\right]+\beta h_{k}^{*}=0
$$

where

$$
\begin{aligned}
v(k, j)= & \frac{1}{\Gamma(2-\alpha)} \\
& \times\left\{\begin{array}{cl}
-k^{1-\alpha}+(k-1)^{1-\alpha} & \text { for } j=0 \\
(k-j+1)^{1-\alpha} & \\
-2(k-j)^{1-\alpha} & \text { for } j=1, \ldots, k-1 \\
+(k-j-1)^{1-\alpha} & \text { for } j=k \\
1 &
\end{array}\right.
\end{aligned}
$$

and

$$
\begin{aligned}
& w(k, i)=\frac{1}{\Gamma(2-\alpha)} \\
& \times\left\{\begin{array}{cl}
(N-k-1)^{1-\alpha} & \\
-(N-k)^{1-\alpha} & \text { for } i=N \\
(i+1-k)^{1-\alpha} & \\
-2(i-k)^{1-\alpha} & \\
+(i-k-1)^{1-\alpha} & \text { for } i=k+1, \ldots, N-1 \\
1 & \text { for } i=k
\end{array}\right.
\end{aligned}
$$

Having Eqn. (8) and its solution in the discrete form Eqn. (16) we can proceed to a brief analysis of the behavior of granular material during the emptying of the silo.

It should be noted that our model Eqn. (8) does not examine the internal structure, e.g. initial solid density, wall roughness, silo shape, solid cohesion, pressure, particle dimensions, etc. Of course, these factors have a significant impact on the mass outflow from the silo. On the other hand, experimental investigations presented by Arevalo et al. [3] demonstrate anomalous behavior of the mass outflow. This means that the dynamics of mass outflow is proportional to a power function of time $\sim t^{\alpha}$. This is contrary to classical models where such dynamics is described by a linear function of time $\sim t$. As explained fractional calculus $[1,3,16,17,31]$ non-integer order $\alpha$ in Eqn. (8) contains the internal structure of processes that mutually influence each. Here we recognize $\alpha$ as the degree of heterogeneity of the mass outflow from the silo. When $\alpha$ tends to unity one can observe regular mass outflow from the silo. On the other hand, $\alpha<1$ means any disturbance of the mass outflow which deviates from the regular/linear mass outflow. Moreover, Eqn. (8) contains another additional parameter $\beta=\lambda^{\alpha}$, where $\lambda$ denotes a delayed outflow of granular material from the silo. In conclusion we can say that the parameters $\alpha$ and $\lambda$ describe the external structure of the outflow. However, according to [3] $\alpha$ parameter contains summary information about the internal structure which has a direct impact on the dynamics of the flow of granular material from the silo. So we cannot estimate $\alpha$ by simplified comparison with experiment done here. Instead we require more complicated experimental data involving more dependencies between parameters describing the internal structure.

\section{Results}

Let us consider some operating states of the silo:

- the mass outflow - as presented by the first case in Sect. 2,

- the irregular outflow, where the capillary force is 10 times less than the actual,

- the funnel outflow, where the capillary force is 4 times less than the actual,

- and the blocade of the silo_-as presented by the second case in Sect. 2.

We have experimental data only for the two extreme cases presented above. Thus, we have results of DEM simulations for all the cases. It should be noted that the results of DEM simulations has been verified by the experimental data only for these two extreme cases-the mass outflow and the silo blocade. Finally, we may have the solution of Eqn. (8) in order approximate the results obtained from the experiment and DEM simulations.

Let us denote $c$ as values of the capillary cohesion. Above states suggest that the capillary cohesion has changed in the interval $c \in\left[0, c_{\max }\right]$, where $c_{\max }$ represents the actual value of the capillary cohesion for which there has been a blockage of the silo. Here we introduced a dimensionless capillary cohesion $c^{*}$, which is defined as $c^{*}=\frac{c}{c_{c \max }}$. Thus, we have $c^{*}=\in[0,1]$. This allows us to identify intermediate states that occur between the mass outflow and the blockade of the silo. Taking into account the changes in static friction coefficient $\mu_{s t}$ determined in Sect. 2 we suggest a simple linear relationship as $\mu_{s t}=0.92-0.11 c^{*}$ being dependent on nondimensionless capillary cohesion $c^{*}$. This expression is suitable in DEM simulations.

Figure 7 presents changes the height loss of granular material over non-dimensional time during the emptying of the silo for all cases determined by the capillary force. We 

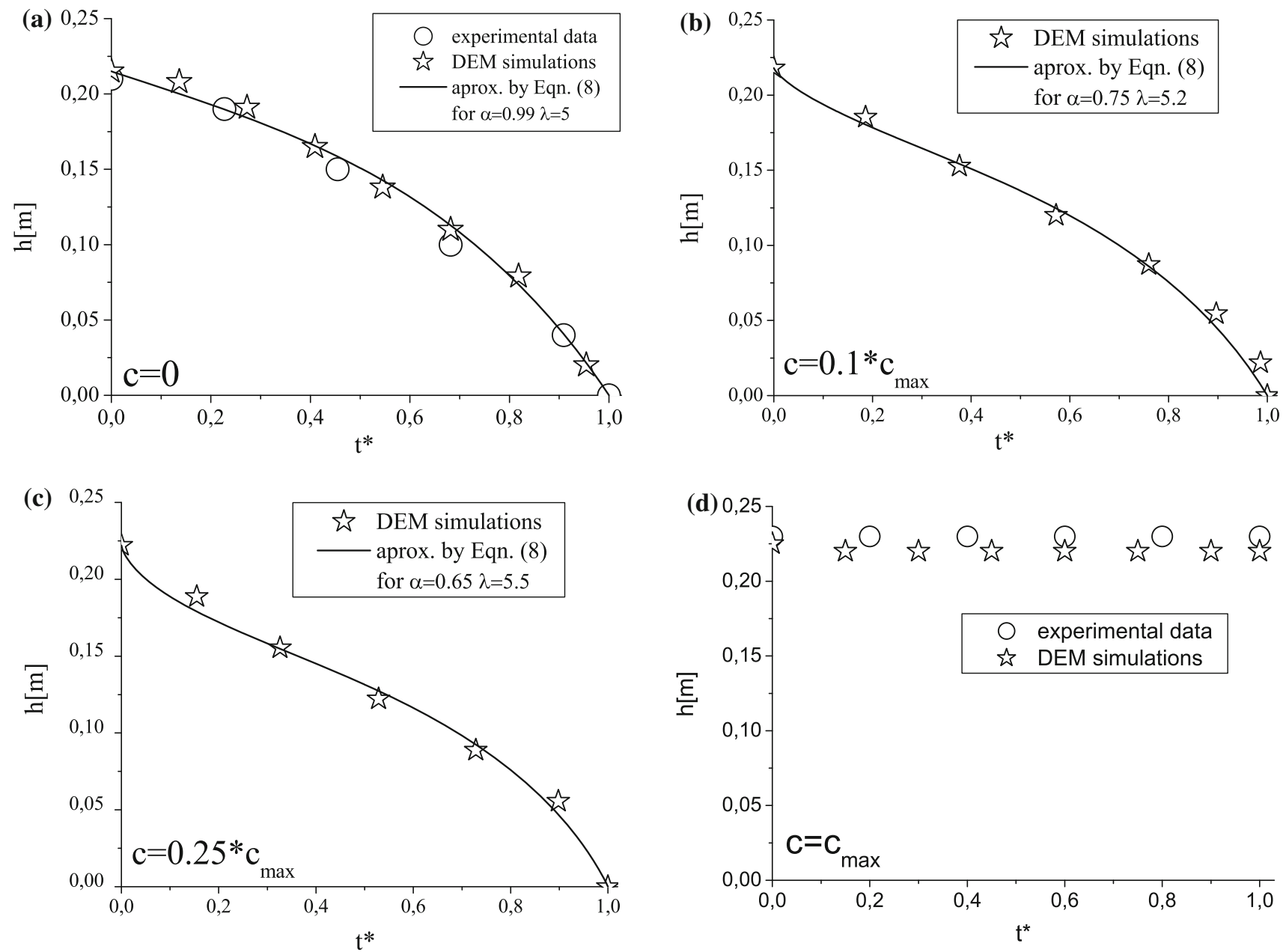

Fig. 7 Operating states of the silo defined by the height loss of granular layer over time: a the mass outflow for $c=0$, $\mathbf{b}$ the irregular outflow for $c=0.10 \cdot c_{\max }, \mathbf{c}$ the irregular outflow for $c=0.25 \cdot c_{\max }$, d outflow is stopped for $c=c_{\max }$
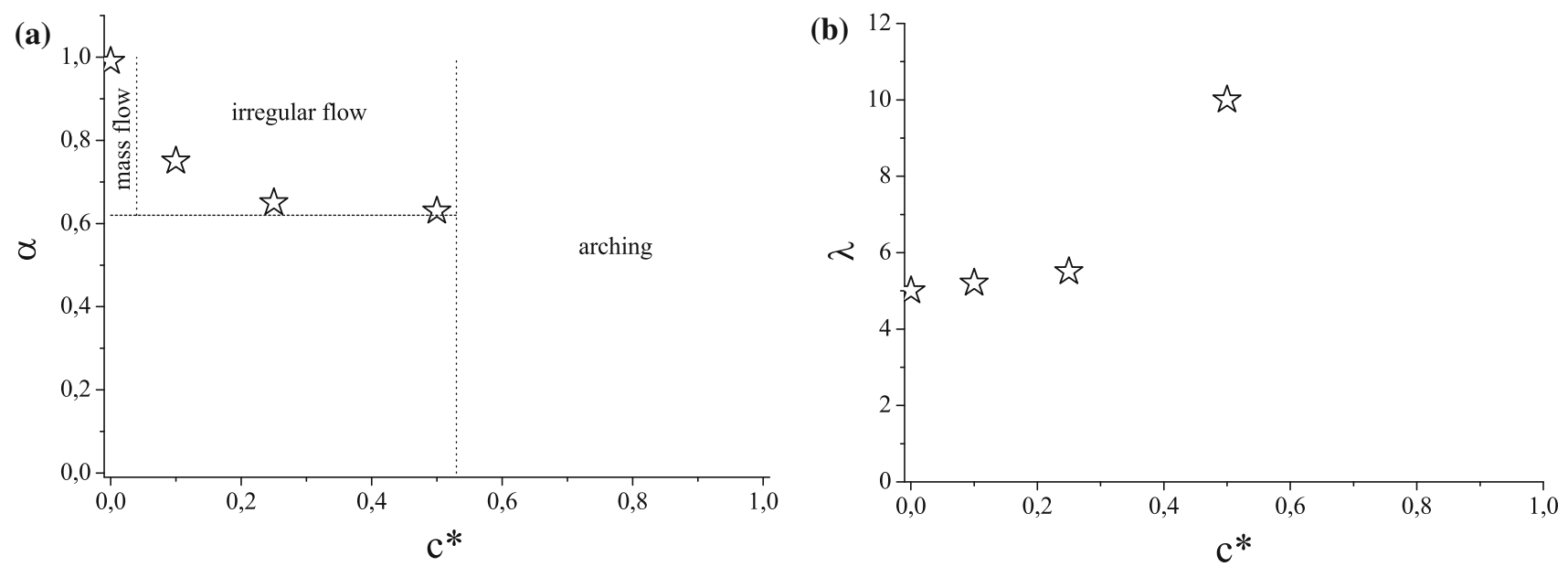

Fig. 8 Change the parameters of: $\mathbf{a} \alpha$ and $\mathbf{b} \lambda$ as a function of dimensionless capillary cohesion 
assumed that $h(t)=h_{\text {bed }}-h^{*}(t)$ where $h^{*}(t)$ is the solution of Eqn. (8), $\beta=\lambda^{1-\alpha}$ is the arbitrary constant in Eqn. (8), $t^{*}=\frac{t}{T}$ indicates non-dimensional time $t^{*} \in[0,1]$ and $T$ is the total emptying time $T \in\{0.88,25.34,31.31,50.00\} \mathrm{s}$. It should be noted that points indicate the experimental data and the DEM simulation results while solid line represents the solution of Eqn. (8) which approximates the above results. Analyzing the behavior of the height loss of the granular layer over the time we can say that in Fig. 7a mass outflow is observed.

Almost linear relationship exists between time and the height loss. In this case we noted $\alpha=0.99$ and $\lambda=5 \frac{1}{s}$ for the approximation given by Eqn. (8). The increase in the value of capillary force, which can be seen in Fig. 7b and $c$, causes irregular outflow in the granular bed. The approximate solution behaves as a function of S-shaped. Then we noticed the lower values of the parameter $\alpha$ in relation to the mass outflow. It should be noted that in the initial phase of the process of emptying is relatively fast loss of the granular layer, which is due to the dominance of gravity. Jamming mechanisms begin to act a little later and then the granular material flows much slower. Middle phase of the outflow of granular material from the silo has a significant impact on the prolongation of the emptying time. In the last phase of emptying, when in the silo is not much granular material, followed by re-acceleration of the outflow. Going towards the maximum values of capillary forces we observed blocking the outflow of granular material. This situation is illustrated in Fig. 7d.

Theoretically, we would have the situation where the parameter $\alpha$ tends to zero. However, for the case $\alpha=0$ differential Eqn. (8) should be changed in an algebraic equation. Therefore, in Fig. 7d has not marked the approximate solution which issues from Eqn. (8). Nevertheless, the analysis of these behaviors will conclude that even before the blockade of the silo shown in Fig. 7d, we received prior information that this would happen.

To explain how it looks like the transition between stable and unstable operation of the silo we trace the changing parameters of Eqn. (8). In particular we are interested some changes in the non-integer order of this equation. Figure 8 shows changes of parameters $\alpha$ and $\lambda$ over the dimensionless capillary cohesion. Analyzing these figures we can conclude that the relationship between capillary cohesion and the parameters existing in Eqn. (8) is particularly evident in Fig. 8a. The figure shows the evolution of parameter $\alpha$ over dimensionless capillary cohesion $c^{*}$. It should be distinguished three phases of the outflow of particles from a silo being dependent on the evolution of the parameter $\alpha$. When $\alpha$ tends to 1 , we observe a mass outflow. If the parameter $\alpha$ decreases then we observed distortions in the operation of the silo. By contrast, when $\alpha$ reaches a value of 0.60 we can say that there had been blocking the outflow of particles from a silo. Next we analyzed the parameter $\lambda$. Figure $8 \mathrm{~b}$ shows the bahavior of $\lambda$ over dimensionless capillary cohesion $c^{*}$. This parameter is called a delayed outflow of granular material from the silo. One can say that this parameter is intensified by the increase of capillary cohesion. It should be noted a sharp jump of $\lambda$ for $c^{*}=0.5$. In this case, there is a block flow of granular material from the silo.

\section{Concluding remarks}

It should highlighted that lack of tools to predict unstable operations of silos is a problem for the industry. In this paper we present a method that allows such a prediction. This method bases on the solution of the fractional oscillator equation, which is an equation over the time, and allows the formulation of initial and final conditions. Our studies show that this equation describes well the change in the time of any variable reflecting processes that occur during the emptying of the silo. Thus, in industrial conditions is sufficient to observe, over the time, increasing mass of granular material which flows from the silo-not necessarily to its total empty. Then, using the solution of Eqn. (8) it should approximate the measurement data. On the basis of values for $\alpha$, we can assess how close we are to regime in which the silo is blocked. However we could not estimate $\alpha$ and $\lambda$ by simple experiment done here. Instead we require more complicated experimental data involving more dependencies between parameters describing the internal structure, e.g. pressure, wall roughness, silo shape, particle dimensions, etc.

Acknowledgments We would like to thank Prof. Bogumil Wrana, Mr. Jan Pietras and Mr. Bartlomiej Czado from Cracow University of Technology for their performace of shear tests and Dr. Mariusz Ciesielski from Czestochowa University of Technology for his fruitful discussion and assistance in carrying out the simulation using DEM.

Open Access This article is distributed under the terms of the Creative Commons Attribution Noncommercial License which permits any noncommercial use, distribution, and reproduction in any medium, provided the original author(s) and source are credited.

\section{References}

1. Agrawal, O.P.: Formulation of Euler-Lagrange equations for fractional variational problems. J. Math. Anal. Appl. 272, 368-379 (2002)

2. Agrawal, O.P.: A general formulation and solution scheme for fractional optimal control problems. Nonlinear Dyn. 38, 323337 (2004)

3. Arevalo, R., Garcimartn, A., Maza, D.: Anomalous diffusion in silo drainage. Eur. Phys. J. E 23, 191-198 (2007)

4. Arnold, P.C., McLean, A.G.: An analytical solution for the stress function at the wall of a converging channel. Powder Technol. 13, 279-285 (1976) 
5. Artoni, R., Santomasoa, A., Canua, P.: Simulation of dense granular flows: dynamics of wall stress in silos. Chem. Eng. Sci. 64, 40404050 (2009)

6. Balevicius, R., Kacianauskas, R., Mroz, Z., Sielamowicz, I.: Microscopic and macroscopic analysis of granular material behaviour in $3 \mathrm{~d}$ flat-bottomed hopper by the discrete element method. Arch. Mech. 59(3), 231-257 (2007)

7. Blaszczyk, T.: Application of Fractional Oscillator Equation for Modeling Memory Effect in Granular Matter. PhD thesis, Czestochowa University of Technology, Czestochowa, Poland (2010) (in polish)

8. Corwin, E., Jaeger, H., Nagel, S.: Structural signature of jamming in granular media. Nature 435, 1075-1078 (2005)

9. Direct/residual shear maschine 27-T2160, operating manual, CONTROLS S.R.L., Italy

10. Drescher, A., Waters, A.J., Rhoades, C.A.: Arching in hoppers: I. Arching theories and bulk material flow properties. Powder Technol. 84, 165-176 (1995)

11. Drescher, A., Waters, A.J., Rhoades, C.A.: Arching in hoppers: II. Arching theories and critical outlet size. Powder Technol. 84, 177183 (1995)

12. Horabik, J., Grochowicz, M.: Determination of yielding parameters of food granular materials. Acta Agrophys. 37, 29-38 (2000)

13. Janssen, H.: Getreidedruck in Silozellen. VDI Zeitschrift 39, 1045 (1895)

14. Jenike, W.: Gravity flow of bulk solids. Utah Eng. Exp. Stn. Bull. 108, 108 (1961)

15. Ketterhagen, W.R., Curtis, J.S., Wassgren, C.R., Hancock, B.C.: Predicting the flow mode from hoppers using the discrete element method. Powder Technol. 195, 1-10 (2009)

16. Kilbas, A.A., Srivastava, H.M., Trujillo, J.J.: Theory and Applications of Fractional Differential Equations. Elsevier, Amsterdam (2006)

17. Klimek, M.: Fractional sequential mechanics-models with symmetric fractional derivative. Czech. J. Phys. 51, 1348-1354 (2001)

18. Klimek, M.: Lagrangean and Hamiltonian fractional sequential mechanics. Czech. J. Phys. 52, 1247-1253 (2002)

19. Klimek, M.: Solutions of Euler-Lagrange equations in fractional mechanics. In: Kielanowski, P., Odzijewicz, A., Schlichenmaier, M., Voronov, T. (eds.) AIP Conference Proceedings 956. XXVI Workshop on Geometrical Methods in Physics, Biaowiea, pp. 7378 (2007)

20. Klimek M.: G-Meijer functions series as solutions for some EulerLagrange equations of fractional mechanics. Conference Proceedings, ENOC, St. Petersburg (2008)
21. Klimek, M.: G-Meijer functions series as solutions for certain fractional variational problem on a finite time interval. J. Eur. Syst. Automatises (JESA) 42, 653-664 (2008)

22. Kozicki, J., Tejchman, J.: Simulations of granular flow in silos with a cellular automata model. Int. J. Storing Handling Process. Powder 13, 267-275 (2001)

23. Kozicki, J., Tejchman, J.: Application of a cellular automaton to simulations of a granular flow in silos. Granular Matter 7, 45-54 (2005)

24. Kuwabara, G., Kono, K.: Restitution coefficient in a collision between two spheres. Jpn. J. Appl. Phys. 26 Part 1, 1230-1233 (1987)

25. Leszczynski, J.S.: Using the fractional interaction law to model the impact dynamics of multiparticle collisions in arbitrary form. Phys. Rev. E 70, 051315 (2004)

26. Longhi, E., Easwar, N.: Large force fluctuations in a flowing granular medium. J. Phys. Rev. Lett. 89, 045501 (2002)

27. Mankoc, C., Janda, A., Arvalo, R., Pastor, J. M., Zuriguel, I., Garcimartn, A., Maza, D.: The flow rate of granular materials through an orifice. Granular Matter 9, 407-414 (2007)

28. Muguruma, Y., Tanaka, T., Tsuji, Y.: Numerical simulation of particulate flow with liquid bridge between particles. Powder Technol. 109, 49-57 (2000)

29. Niedostatkiewicz, M., Tejchman, J.: Experimental and theoretical studies on resonance dynamic effects during silo flow. Powder Handling Process. Trans. Tech. Publ. 15, 36-42 (2003)

30. Nguyen, T.V., Brennen, C.E., Sabersky, R.H.: Funnel flow in hoppers. J. Appl. Mech. 47(4), 729-735 (1980)

31. Riewe, F.: Nonconservative Lagrangian and Hamiltonian mechanics. Phys. Rev. E 53, 1890-1899 (1996)

32. Riewe, F.: Mechanics with fractional derivatives. Phys. Rev. E 55, 3581-3592 (1997)

33. Samadani, A., Kudrolli, A.: Angle of repose and segregation in cohesive granular matter. Phys. Rev. E 64, 051301 (2001)

34. Schulze, D.: Powders and Bulk Solids-Behavior, Characterization, Storage and Flow. Springer, Berlin (2007)

35. Stasiak, M., Molenda, M.: Direct shear testing of flowability of food powders. Res. Agric. Eng. 50(1), 6-10 (2004)

36. Zuriguel, I., Pugnaloni, L.A., Garcimartn, A., Maza, D.: Jamming during the discharge of grains from a silo described as a percolating transition. Phys. Rev. E 68, 030301 (2003)

37. Zuriguel, I., Garcimartn, A., Maza, D., Pugnaloni, L.A., Pastor, J.M.: Jamming during the discharge of granular matter from a silo. Phys. Rev. E 71, 051303 (2005) 